\title{
Solving a Class of Singular Two-Point Boundary Value Problems Using New Modified Decomposition Method
}

\author{
Randhir Singh and Jitendra Kumar \\ Department of Mathematics, Indian Institute of Technology Kharagpur, Kharagpur 721302, India \\ Correspondence should be addressed to Randhir Singh; randhir.math@gmail.com
}

Received 10 November 2012; Accepted 5 January 2013

Academic Editors: T. Allahviranloo, L. S. Heath, E. Weber, and J. G. Zhou

Copyright ( 2013 R. Singh and J. Kumar. This is an open access article distributed under the Creative Commons Attribution License, which permits unrestricted use, distribution, and reproduction in any medium, provided the original work is properly cited.

\begin{abstract}
We introduce an effective methodology for solving a class of linear as well as nonlinear singular two-point boundary value problems. This methodology is based on a modification of Adomian decomposition method (ADM) and a new two fold integral operator. We use all the boundary conditions to derive an integral equation before establishing the recursive scheme for the solution components of solution. Thus, we develop modified recursive scheme without any undetermined coefficients while computing the successive solution components. This modification also avoids solving a sequence of nonlinear algebraic or transcendental equations for the undetermined coefficients. However, most of earlier recursive schemes using ADM do require computation of undetermined coefficients. The approximate solution is obtained in the form of series with easily calculable components. Numerical examples are included to demonstrate the accuracy, applicability, and generality of the present technique. The results reveal that the method is very effective, straightforward, and simple.
\end{abstract}

\section{Introduction}

We consider the following class of singular two-point boundary value problems [1-5]:

$$
\left(x^{\alpha} y^{\prime}\right)^{\prime}=f(x, y), \quad 0<x \leq 1,
$$

subject to the boundary conditions

$$
y(0)=d, \quad a y(1)+b y^{\prime}(1)=c,
$$

where $a>0, b, c$, and $d$ are any finite constants and $0 \leq \alpha<1$. We assume that, for $(x, y) \in\{[0,1] \times \mathbb{R}\}$, the function $f(x, y)$ and $\partial f / \partial y$ are continuous and $\partial f / \partial y \geq 0$. In particular, the problem (1) arises very frequently in applied sciences and in physiological studies, for example, in the study of steady-state oxygen diffusion in a spherical cell with Michaelis-Menten uptake kinetics [6] and distribution of heat sources in the human head [7]. In particular, when $\alpha=0$ and $f(x, y)=$ $x^{-1 / 2} y^{3 / 2}(1)$ is known as Thomas-Fermi equation [8], given the singular equation

$$
y^{\prime \prime}=x^{-1 / 2} y^{3 / 2} .
$$

Recently, there has been much interest in the study of singular two-point boundary value problems of type (1), (see, e.g., $[1-5,8-15])$ and many of the references therein. The main difficulty of problem (1) is that the singularity behavior occurs at $x=0$. A lot of methods have been applied to tackle this singular boundary value problem. In [9], a standard three-point finite difference scheme was considered with uniform mesh for the solution of problem for $\alpha \in(0,1)$. In $[1,5]$, the finite difference methods were used to obtain the numerical solutions. In [4], a numerical method based on Green's function was used to obtain numerical solution of the same problem. A novel approach that combines a modified decomposition method with the cubic B-spline collocation technique is presented in [14] to obtain approximate solution with high accuracy. Recently, in [15], a new modified decomposition method was applied to tackle these problems. These numerical methods have many advantages, but a huge amount of computational work is required to obtain accurate numerical solution especially for nonlinear problems.

1.1. Adomian Decomposition Method (ADM). In this subsection, we briefly describe standard ADM or MADM for nonlinear second-order equation. 
Recently, many researchers $[3,8,12-24]$ have shown interest in the study of ADM for different scientific models. Adomian [20] asserted that the ADM provides an efficient and computationally suitable method for generating approximate series solution for a large class of differential equations.

According to Wazwaz [24], (1) in operator form is given as

$$
L y=x^{-\alpha} f(x, y),
$$

where $L$ is a linear differential operator defined as

$$
L=x^{-\alpha} \frac{d}{d x}\left(x^{\alpha} \frac{d}{d x}\right) .
$$

The inverse operator $L^{-1}$ is given as (see [24])

$$
L^{-1}(\cdot)=\int_{0}^{x} x^{-\alpha} \int_{0}^{x} x^{\alpha}(\cdot) d x d x
$$

Operating the inverse linear operator $L^{-1}(\cdot)$ on both sides of (4) yields

$$
y=y(0)+x y^{\prime}(0)+L^{-1}\left[x^{-\alpha} f(x, y)\right] .
$$

Next, we decompose the solution $y$ and the nonlinear function $N(y) \equiv f(x, y)$ by an infinite series as

$$
y=\sum_{n=0}^{\infty} y_{n}, \quad N(y)=\sum_{n=0}^{\infty} A_{n},
$$

where $A_{n}$ are Adomian's polynomials that can be constructed for various classes of nonlinear functions with the formula given in [17] as

$$
A_{n}=\frac{1}{n !} \frac{d^{n}}{d \lambda^{n}}\left[N\left(\sum_{k=0}^{\infty} y_{k} \lambda^{k}\right)\right]_{\lambda=0}, \quad n=0,1,2, \ldots
$$

Substituting the series (8) into (7), we obtain

$$
\sum_{n=0}^{\infty} y_{n}=y(0)+x y^{\prime}(0)+L^{-1}\left[x^{-\alpha} \sum_{n=0}^{\infty} A_{n}\right] \text {. }
$$

Upon matching both sides of (10), the standard ADM is given by

$$
\begin{gathered}
y_{0}=y(0)+x y^{\prime}(0), \\
y_{k+1}=L^{-1}\left[x^{-\alpha} A_{k}\right], \quad k \geq 0
\end{gathered}
$$

and modified ADM is given as

$$
\begin{gathered}
y_{0}=y(0), \\
y_{1}=x y^{\prime}(0)+L^{-1}\left[x^{-\alpha} A_{0}\right], \\
y_{k+1}=L^{-1}\left[x^{-\alpha} A_{k}\right], \quad k \geq 0 .
\end{gathered}
$$

Having determined the components $y_{n}, n \geq 0$ recurrently, the series solution of $y$ follows immediately with the undetermined coefficient $y^{\prime}(0)=\eta_{1}$, is as yet to be determined
$[12,21,22]$. For numerical purpose, the $n$-term truncated series

$$
\phi_{n}(x)=\sum_{m=0}^{n} y_{m}(x)
$$

may be used to give the approximate solution.

The decomposition method has been applied to solve nonlinear boundary value problems for ordinary differential equations by several researchers [3, 12-16, 18, 19, 21-23]. Solving such problems using standard ADM or MADM is always a computationally involved task as it requires the computation of undetermined coefficients in a sequence of nonlinear algebraic equations which increases the computational work (see $[3,14,15,21-23])$. A major disadvantage of the earlier methods for solving nonlinear BVPs is that we need to solve a sequence of growingly higher-order polynomials or more difficult transcendental equations [3, 21, 23]. For example, consider nonlinear equation

$$
\begin{aligned}
& y^{\prime \prime}=e^{y}, \quad 0 \leq x \leq 1, \\
& y(0)=0, \quad y(1)=0 .
\end{aligned}
$$

Applying the standard ADM (11) with initial guess $y_{0}=\eta_{1} x$, where $y^{\prime}(0)=\eta_{1}$ to above problem (14), we obtain the solution components as

$$
\begin{aligned}
& y_{0}=\eta_{1} x \\
& y_{1}=\frac{\left(-1+e^{\eta_{1} x}-\eta_{1} x\right)}{\eta_{1}^{2}}, \\
& y_{2}=\frac{\left(-5+e^{2 \eta_{1} x}-2 \eta_{1} x+e^{\eta_{1} x}\left(4-4 \eta_{1} x\right)\right)}{4 \eta_{1}^{4}}, \\
& y_{3}=\left(-22+e^{3 \eta_{1} x}-6 \eta_{1} x+e^{2 \eta_{1} x}\left(6-6 \eta_{1} x\right)\right. \\
& \left.\quad+3 e^{\eta_{1} x}\left(5+2 \eta_{1} x\left(-3+\eta_{1} x\right)\right)\right) \times\left(12 \eta_{1}^{6}\right)^{-1}
\end{aligned}
$$

and $n$-term approximate solution $\phi_{n}(x)=\sum_{m=0}^{n} y_{m}$ as

$$
\begin{aligned}
\phi_{1}(x) & =\eta_{1} x+\frac{\left(-1+e^{\eta_{1} x}-\eta_{1} x\right)}{\eta_{1}^{2}}, \\
\phi_{2}(x)=\eta_{1} x+\frac{\left(-1+e^{\eta_{1} x}-\eta_{1} x\right)}{\eta_{1}^{2}} & \\
+ & \frac{\left(-5+e^{2 \eta_{1} x}-2 \eta_{1} x+e^{\eta_{1} x}\left(4-4 \eta_{1} x\right)\right)}{4 \eta_{1}^{4}},
\end{aligned}
$$




$$
\begin{aligned}
\phi_{3}(x)= & \eta_{1} x+\frac{\left(-1+e^{\eta_{1} x}-\eta_{1} x\right)}{\eta_{1}^{2}} \\
& +\frac{\left(-5+e^{2 \eta_{1} x}-2 \eta_{1} x+e^{\eta_{1} x}\left(4-4 \eta_{1} x\right)\right)}{4 \eta_{1}^{4}} \\
& +\left(-22+e^{3 \eta_{1} x}-6 \eta_{1} x+e^{2 \eta_{1} x}\left(6-6 \eta_{1} x\right)\right. \\
& \left.+3 e^{\eta_{1} x}\left(5+2 \eta_{1} x\left(-3+\eta_{1} x\right)\right)\right) \times\left(12 \eta_{1}^{6}\right)^{-1}
\end{aligned}
$$

Note that the above calculations become more and more complicated and cannot lead to exact results for the other approximations. To obtain approximate solution, the boundary condition $y(1)=0$ is imposed into $\phi_{3}$, and solving for $\eta_{1}$, we can obtain the approximation. However, solving this transcendental equation for $\eta_{1}$ requires additional computational work, and $\eta_{1}$ may not be uniquely determined (see $[3,15,21,23])$.

In order to avoid solving such nonlinear algebraic or transcendental equations for two-point boundary value problems, in [16], extended ADM was introduced for nonsingular problems with Dirichlet boundary conditions. The method proposed in [13] was based on the new modification of ADM. Recently, a special modified inverse linear integral operators for higher-order boundary value problem was given in [25].

In this paper, a new modification of the ADM is proposed to overcome the difficulties occurred in the standard ADM or MADM for solving nonlinear singular boundary value problems (1). To obtain a new modified decomposition method, we operate our proposed twofold integral operator on both sides of (1) and use all the boundary conditions to establish the recursion scheme for the solution components of the solution. We propose a modified recursion scheme which does not require the computation of unknown constants, that is, without solving a sequence of growingly higher-order polynomial or difficult transcendental equations to obtain unknown constant $[3,14,15,21,23]$. The main advantage of our proposed method is that it provides a direct recursive scheme for solving the singular boundary value problem.

The rest of the paper is organized as follows. In Section 2, the description of the proposed recursive scheme based on new definition of differential operator and its inverse operator is given. In Section 3, we illustrate our method with numerical results along with the graphical representation. In Section 4, the conclusion is given.

\section{New Modified Decomposition Method}

In this section, we propose a new modified ADM based on two-fold integral operator for solving linear as well as nonlinear singular two-point boundary value problems.

To overcome the singular behavior at $x=0$, we again rewrite (1)

$$
\left(x^{\alpha} y^{\prime}\right)^{\prime}=f(x, y), \quad 0<x \leq 1
$$

which can be rewritten in operator form as

$$
L y=f(x, y) \text {, }
$$

where $L y \equiv\left(x^{\alpha} y^{\prime}\right)^{\prime}$ is the linear differential operator.

Twofold integral operator $L^{-1}(\cdot)$ regarded as the inverse operator of $L(\cdot)$ is proposed as

$$
L^{-1}(\cdot) \equiv \int_{0}^{x} x^{-\alpha}\left(\int_{x}^{1}(\cdot) d x\right) d x .
$$

To establish a new modified recursive scheme, we operate $L^{-1}(\cdot)$ on the left-hand side of (17) and use the boundary condition $y(0)=d$, which yields

$$
\begin{aligned}
& L^{-1}\left[\left(x^{\alpha} y^{\prime}\right)^{\prime}\right] \\
& \quad=\int_{0}^{x} x^{-\alpha}\left(\int_{x}^{1}\left(x^{\alpha} y^{\prime}\right)^{\prime} d x\right) d x, \\
& =\int_{0}^{x} x^{-\alpha}\left(\int_{x}^{1}\left(x^{\alpha} y^{\prime \prime}+\alpha x^{\alpha-1} y^{\prime}\right) d x\right) d x, \\
& =\int_{0}^{x} x^{-\alpha}\left(y^{\prime}(1)-x^{\alpha} y^{\prime}(x)-\int_{x}^{1} \alpha x^{\alpha-1} y^{\prime} d x\right. \\
& \quad=c_{1} \int_{0}^{x} x^{-\alpha} d x-y(x)+d, \\
& L^{-1}\left[\left(x^{-\alpha} y^{\prime}\right)^{\prime}\right]=c_{1} \frac{x^{1-\alpha}}{1-\alpha}-y(x)+d,
\end{aligned}
$$

where $y^{\prime}(1)=c_{1}$.

Now, we again operate the inverse operator $L^{-1}(\cdot)$ on both sides of (17) and use (20), which gives

$$
y(x)=d+\frac{c_{1} x^{1-\alpha}}{1-\alpha}-\int_{0}^{x} x^{-\alpha}\left(\int_{x}^{1} f(x, y) d x\right) d x
$$

Imposing the boundary condition $a y(1)+b y^{\prime}(1)=c$ into (21), we get

$$
\begin{aligned}
c_{1}= & \frac{(c-a d)(1-\alpha)}{a+b(1-\alpha)}+\frac{a(1-\alpha)}{a+b(1-\alpha)} \\
& \times\left[\int_{0}^{1} x^{-\alpha}\left(\int_{x}^{1} f(x, y) d x\right) d x\right] .
\end{aligned}
$$

For simplicity, we set $\left[L^{-1} f(x, y)\right]_{x=1}=\int_{0}^{1} x^{-\alpha}\left(\int_{x}^{1} f(x\right.$, y) $d x) d x$.

Substituting the value of $c_{1}$ into (21), we obtain

$$
\begin{aligned}
y(x)= & d+\frac{(c-a d) x^{1-\alpha}}{a+b(1-\alpha)}+\frac{a x^{1-\alpha}}{a+b(1-\alpha)} \\
& \times\left[L^{-1} f(x, y)\right]_{x=1}-\left[L^{-1} f(x, y)\right] .
\end{aligned}
$$


Note that the right-hand side of (23) does not involve any undetermined coefficients.

Next, we decompose the solution $y(x)$ by series as

$$
y=\sum_{n=0}^{\infty} y_{n}
$$

and the nonlinear function $f(x, y)$ is decomposed by series as

$$
f(x, y)=\sum_{n=0}^{\infty} A_{n}\left(y_{0}, y_{1}, \ldots, y_{n}\right),
$$

where $A_{n}\left(y_{0}, y_{1}, \ldots, y_{n}\right)$ are Adomian's polynomials [17].

Substituting the series (24) and (25) into (23), we obtain

$$
\begin{aligned}
\sum_{n=0}^{\infty} y_{n}= & +\frac{(c-a d) x^{1-\alpha}}{a+b(1-\alpha)}+\frac{a x^{1-\alpha}}{a+b(1-\alpha)} \\
& \times\left[L^{-1} \sum_{n=0}^{\infty} A_{n}\right]_{x=1}-\left[L^{-1} \sum_{n=0}^{\infty} A_{n}\right] .
\end{aligned}
$$

Upon matching both sides of (26), the new modified decomposition method is given by

$$
\begin{aligned}
y_{0}= & d, \\
y_{1}= & \frac{(c-a d) x^{1-\alpha}}{a+b(1-\alpha)}+\frac{a x^{1-\alpha}}{a+b(1-\alpha)} \\
& \times\left[L^{-1} A_{0}\right]_{x=1}-\left[L^{-1} A_{0}\right], \\
y_{n+1}= & \frac{a x^{1-\alpha}}{a+b(1-\alpha)}\left[L^{-1} A_{n}\right]_{x=1} \\
& -\left[L^{-1} A_{n}\right], \quad n \geq 1, \text { or } \\
y_{n+1}= & \frac{a x^{1-\alpha}}{a+b(1-\alpha)} \int_{0}^{1} x^{-\alpha} \int_{x}^{1} A_{n} d x d x \\
& -\int_{0}^{x} x^{-\alpha} \int_{x}^{1} A_{n} d x d x, \quad n \geq 1 .
\end{aligned}
$$

It should be noted that the new modified recursive scheme (27) requires no additional computational work as it does not involve any unknown constant. This slight change plays a major role in minimizing the computational work. This modification also avoids solving a sequence of nonlinear algebraic or transcendental equations for the undetermined coefficients with multiple roots, which is required to complete the calculation of the solution by several earlier modified recursion schemes using the ADM or MADM [3, 14, 22, 23]. The above new modified scheme (27) gives the complete determination of solution components $y_{n}$ of solution $y$, and hence, the approximate series solution $\psi_{n}(x)$ can be obtained by adding the solution components $y_{0}, y_{1}, \ldots, y_{n}$. However, for numerical purpose, the truncated $n$-term approximate series solution is given by

$$
\psi_{n}(x)=\sum_{m=0}^{n} y_{m}(x),
$$

and the limiting value of sequence $\psi_{n}(x)$ converges to exact solution $y$. We remark that the convergence of Adomian decomposition method for differential and integral equations has already been established by many authors [26-28].

\section{Numerical Illustrations and Discussions}

In this section, the new modified ADM (27) is implemented for tackling singular boundary value problems (1). We demonstrate the effectiveness of this method with five examples. All the numerical results obtained by the proposed method are compared with known results. Furthermore, the maximum error functions $E_{n}(x)$ and approximate solutions $\psi_{n}$ are plotted.

Example 1. Consider the nonlinear singular two-point boundary value problem [10]

$$
\begin{gathered}
\left(x^{\alpha} y^{\prime}\right)^{\prime}=\beta x^{\alpha+\beta-2} e^{y}\left(\beta x^{\beta} e^{y}-\alpha-\beta+1\right), \\
0<x \leq 1, \\
y(0)=-\ln (4), \quad y(1)=-\ln (5),
\end{gathered}
$$

with exact solution $y(x)=\ln \left(1 /\left(4+x^{\beta}\right)\right)$.

We apply scheme (27) to (29), where $0 \leq \alpha<1$ and $a=1$, $b=0, c=-\ln (5)$, and $d=-\ln (4)$, scheme (27) for (29) can be read as follows

$$
\begin{aligned}
y_{0}= & -\ln (4), \\
y_{1}= & (\ln 4-\ln 5) x^{1-\alpha}+x^{1-\alpha} \int_{0}^{1} x^{-\alpha} \int_{x}^{1} x^{\alpha+\beta-2} A_{0} d x d x \\
& -\int_{0}^{x} x^{-\alpha} \int_{x}^{1} x^{\alpha+\beta-2} A_{0} d x d x \\
y_{n+1}= & x^{1-\alpha} \int_{0}^{1} x^{-\alpha} \int_{x}^{1} x^{\alpha+\beta-2} A_{n} d x d x \\
& \quad-\int_{0}^{x} x^{-\alpha} \int_{x}^{1} x^{\alpha+\beta-2} A_{n} d x d x, \quad n \geq 1 .
\end{aligned}
$$

The Adomian's polynomials for nonlinear function $f(x, y)=$ $\beta\left(\beta x^{\beta} e^{2 y}-e^{y}(\alpha+\beta-1)\right)$ with $y_{0}=-\ln (4)$ are obtained by formula (9) given as

$$
\begin{aligned}
A_{0}= & \beta\left[0.0625 \beta x^{\beta}+0.25(1-\alpha-\beta)\right], \\
A_{1}= & \beta\left[0.125 \beta x^{\beta}+0.25(1-\alpha-\beta)\right] y_{1}, \\
A_{2}= & \beta\left[0.125 \beta x^{\beta}+0.25(1-\alpha-\beta)\right] y_{2} \\
& +\beta\left[0.25 \beta x^{\beta}+0.25(1-\alpha-\beta)\right] \frac{y_{1}^{2}}{2},
\end{aligned}
$$

For the demonstration purpose, we chose some specific values of $\alpha$ and $\beta$. 


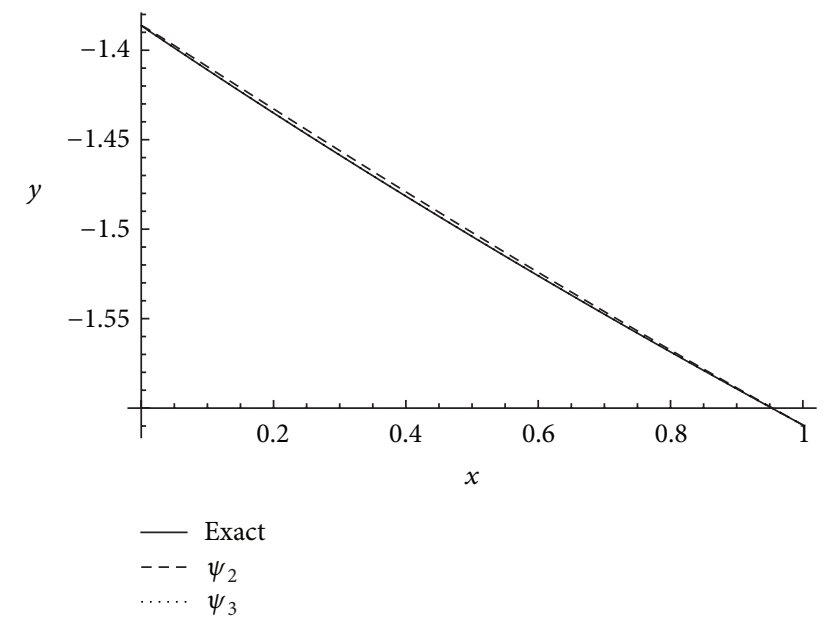

FIGURE 1: Comparison of exact solution $y$ and approximate solutions $\psi_{n}, n=2,3$, of Example 1 when $\alpha=0.5, \beta=1$.

For $\alpha=0.25, \beta=1$, by using (30) and (31), we obtain the successive solution components $y_{n}$ of solution $y(x)$ as

$$
\begin{aligned}
y_{0}= & -1.38629, \\
y_{1}= & 0.0018564 x^{0.75}-0.25 x+0.025 x^{2}, \\
y_{2}= & -0.0016051 x^{0.75}-0.000066301 x^{1.75} \\
& +0.00625 x^{2}+0.00004219 x^{2.75} \\
& -0.0048611 x^{3}+0.00024038 x^{4},
\end{aligned}
$$

For $\alpha=0.5, \beta=1$, we again use (30) and (31) to obtain the successive solution components as

$$
\begin{aligned}
y_{0}= & -1.38629, \\
y_{1}= & +0.00602312 x^{0.5}-0.25 x+0.0208333 x^{2}, \\
y_{2}= & -0.00573744 x^{0.5}-0.000501926 x^{1.5} \\
& +0.0104167 x^{2}+0.00015057 x^{2.5} \\
& -0.00451389 x^{3}+0.000186012 x^{4}
\end{aligned}
$$

Remark 2. In a similar manner, we can calculate any solution components $y_{n}$ of solution for different values $0 \leq \alpha<1$ and $\beta>0$. For simplicity, we have not listed all the solution components $y_{n}$ for different values of $\alpha$ and $\beta$, but we have calculated the first ten solution components.

Now, we define error functions as $E_{n}(x)=\left|\psi_{n}(x)-y(x)\right|$ and the maximum absolute error is given as

$$
E^{(n)}=\max _{0<x \leq 1} E_{n}(x) .
$$

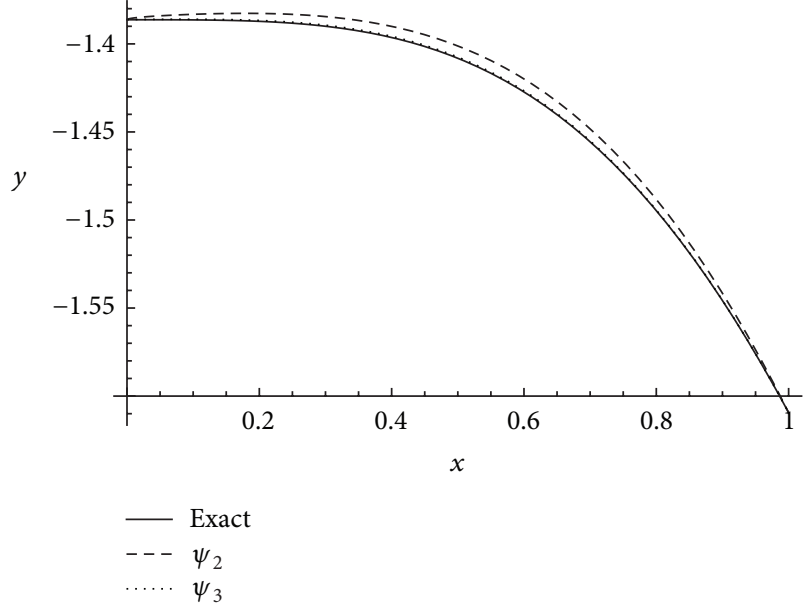

FIgURE 2: Comparison of exact solution $y$ and approximate solutions $\psi_{n}, n=2,3$, of Example 1 when $\alpha=0.5, \beta=3.5$.

TABLE 1: Maximum error of Example 1, when $\beta=1$.

\begin{tabular}{lccc}
\hline$\alpha$ & $E^{(5)}$ & $E^{(8)}$ & $E^{(10)}$ \\
\hline 0.25 & $2.24869 \times 10^{-6}$ & $3.14283 \times 10^{-9}$ & $3.90384 \times 10^{-10}$ \\
0.5 & $1.26007 \times 10^{-6}$ & $1.24544 \times 10^{-9}$ & $1.51616 \times 10^{-11}$ \\
0.75 & $7.66693 \times 10^{-7}$ & $4.07955 \times 10^{-10}$ & $9.72399 \times 10^{-12}$ \\
\hline
\end{tabular}

We plot approximate solutions $\psi_{n}$, for $n=2,3$, and exact solution $y$ in Figures 1 and 2 for different values of $\alpha$ and $\beta$. The error functions $E_{n}(x)$, for $n=3,4,5$, are plotted in Figures 3 and 4 . In addition, the maximum absolute errors $E^{(n)}$, for $n=5,8,10$, are listed in Tables 1 and 2 .

Example 3. Consider the nonlinear singular two-point boundary value problem

$$
\begin{gathered}
\left(x^{1 / 2} y^{\prime}\right)^{\prime}=\frac{3}{16}\left(-4+5 x^{3 / 2}\right) y^{5}, \quad 0<x<1, \\
y(0)=1, \quad y(1)=\sqrt{\frac{1}{2}}=0.7071067812,
\end{gathered}
$$

with exact solution $y(x)=\sqrt{1 /\left(1+x^{3 / 2}\right)}$.

On applying scheme (27) to (35) where $a=1, b=0$, $c=0.7071067812$, and $d=1$, scheme (27) for (35) becomes as follows:

$$
\begin{aligned}
y_{0}= & 1 \\
y_{1}= & 0.2928932 x^{1 / 2}+x^{1 / 2} \int_{0}^{1} x^{-1 / 2} \int_{x}^{1} g(x) A_{0} d x d x \\
& -\int_{0}^{x} x^{-1 / 2} \int_{x}^{1} g(x) A_{0} d x d x, \\
y_{n+1}= & x^{1 / 2} \int_{0}^{1} x^{-1 / 2} \int_{x}^{1} g(x) A_{n} d x d x \\
& \quad-\int_{0}^{x} x^{-1 / 2} \int_{x}^{1} g(x) A_{n} d x d x, \quad n \geq 1,
\end{aligned}
$$

where $g(x)=(3 / 16)\left(-4+5 x^{3 / 2}\right)$. 


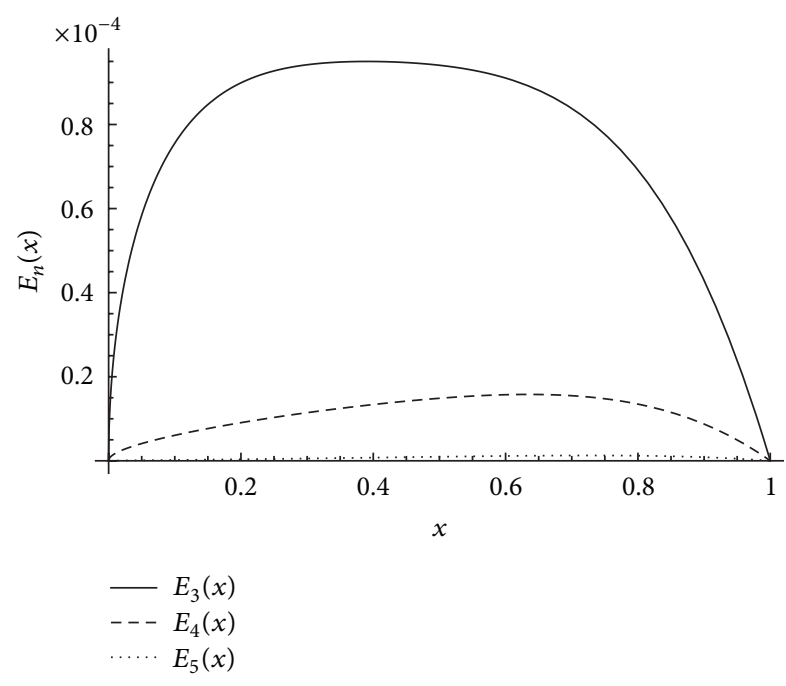

FIgURE 3: Error functions $E_{n}, n=3,4,5$, of Example 1 when $\alpha=$ $0.5, \beta=1$.

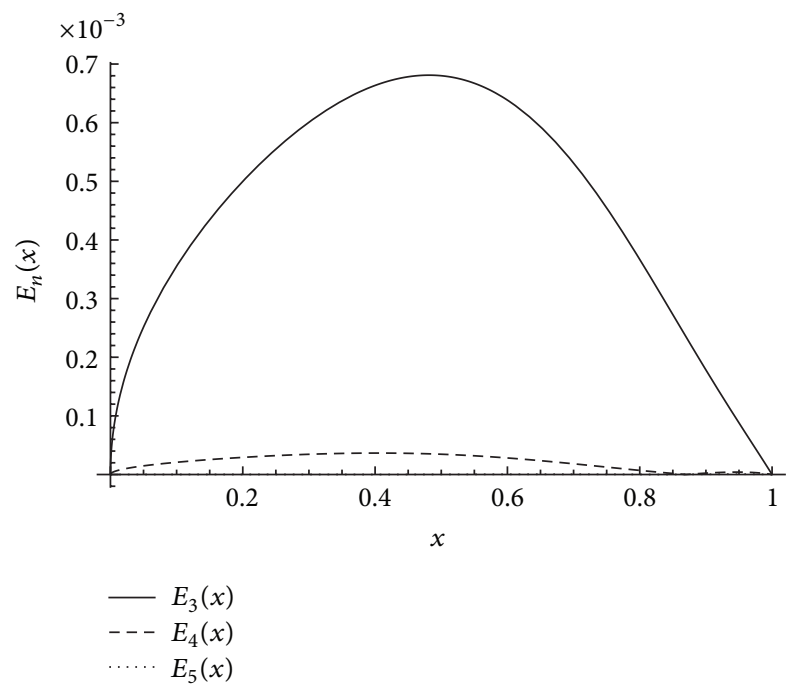

Figure 4: Error functions $E_{n}, n=3,4,5$ of Example 1 when $\alpha=$ $0.5, \beta=3.5$.

As given above, the Adomian polynomials for nonlinear function $f(y)=y^{5}$ with $y_{0}=1$ are given by

$$
\begin{aligned}
& A_{0}=1, \\
& A_{1}=5 y_{1}, \\
& A_{2}=10 y_{1}^{2}+5 y_{2}, \\
& A_{3}=10 y_{1}^{3}+20 y_{1} y_{2}+5 y_{3},
\end{aligned}
$$

TABLE 2: Maximum error of Example 1, when $\beta=3.5$.

\begin{tabular}{lccc}
\hline$\alpha$ & $E^{(5)}$ & $E^{(8)}$ & $E^{(10)}$ \\
\hline 0.25 & $8.41020 \times 10^{-7}$ & $7.75054 \times 10^{-10}$ & $2.30935 \times 10^{-11}$ \\
0.5 & $3.97263 \times 10^{-7}$ & $5.87251 \times 10^{-9}$ & $7.81719 \times 10^{-11}$ \\
0.75 & $3.42314 \times 10^{-6}$ & $2.78721 \times 10^{-8}$ & $9.19921 \times 10^{-11}$ \\
\hline
\end{tabular}

TABle 3: Maximum error of Example 3.

\begin{tabular}{lccc}
\hline$n$ & 5 & 8 & 10 \\
\hline$E^{(n)}$ & $7.385093 \times 10^{-4}$ & $2.3947849 \times 10^{-5}$ & $9.7489281 \times 10^{-6}$ \\
\hline
\end{tabular}

Using (36) and (37), we have the successive solution components as

$$
\begin{aligned}
y_{0}= & 1, \\
y_{1}= & 0.0821068 x^{0.5}-0.5 x^{1.5}+0.125 x^{3}, \\
y_{2}= & -0.045527 x^{0.5}-0.102633 x^{2} \\
& +0.25 x^{3}+0.0366548 x^{3.5} \\
& -0.15625 x^{4.5}+0.0177557 x^{6},
\end{aligned}
$$

In order to verify how close the approximate solutions are to the exact solution, we plot approximate solutions $\psi_{n}, n=$ $1,2,4$, and exact solution $y$ in Figure 5 and conclude that the approximate solution $\psi_{4}$ is very close to exact solution $y$. In Figure 6, the error functions $E_{n}(x)$, for $n=5,6,7$, are plotted. From Figure 6 we can see that when $n$ increases, the error decreases. In addition, the maximum absolute errors $E^{(n)}$, for $n=5,8,10$, are listed in Table 3 .

Example 4. Consider the nonlinear singular two-point boundary value problem $[15,23]$

$$
\begin{aligned}
y^{\prime \prime}+\frac{0.5}{x} y^{\prime} & =\left(0.5 e^{y}-e^{2 y}\right), \quad 0<x<1 \\
y(0) & =\ln (2), \quad y(1)=0,
\end{aligned}
$$

with exact solution $y(x)=\ln \left[2 /\left(x^{2}+1\right)\right]$.

We apply scheme (27) to (39), where $\alpha=0.5$ and $a=1$, $b=c=0$, and $d=\ln (2)$, scheme (27) for (39) becomes

$$
\begin{aligned}
y_{0}= & \ln (2) \\
y_{1}= & -\ln (2) x^{1 / 2}+x^{1 / 2} \int_{0}^{1} x^{-1 / 2} \int_{x}^{1} x^{1 / 2} A_{0} d x d x \\
& -\int_{0}^{x} x^{-1 / 2} \int_{x}^{1} x^{1 / 2} A_{0} d x d x \\
y_{n+1}= & x^{1 / 2} \int_{0}^{1} x^{-1 / 2} \int_{x}^{1} x^{1 / 2} A_{n} d x d x \\
& \quad-\int_{0}^{x} x^{-1 / 2} \int_{x}^{1} x^{1 / 2} A_{n} d x d x, \quad n \geq 1
\end{aligned}
$$




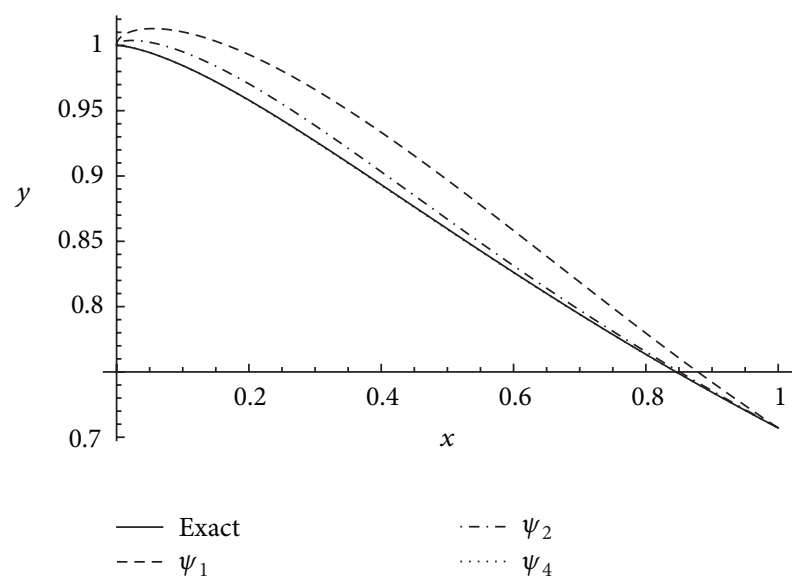

Figure 5: Comparison of exact solution $y$ and approximate solutions $\psi_{n}, n=1,2,4$ of Example 3.

Proceeding as before, the Adomian's polynomials for $f(y)=$ $\left(0.5 e^{y}-e^{2 y}\right)$ with $y_{0}=\ln (2)$ are given as

$$
\begin{aligned}
& A_{0}=-3, \\
& A_{1}=-7 y_{1}, \\
& A_{2}=-7.5 y_{1}^{2}-7 y_{2}, \\
& A_{3}=-5.16667 y_{1}^{3}-15 y_{1} y_{2}-7 y_{3},
\end{aligned}
$$

Making use of (40) and (41), we have successive solution components $y_{n}$ of solution $y(x)$ as

$$
\begin{aligned}
y_{0}= & \ln (2), \\
y_{1}= & 0.0211385 x^{0.5}-x^{2}+0.285714 x^{4}, \\
y_{2}= & 0.0193055 x^{0.5}-0.0126831 x^{2.5} \\
& \quad+0.214286 x^{4}+0.0093949 x^{4.5} \\
& \quad 0.268398 x^{6}+0.0380952 x^{8}
\end{aligned}
$$

To verify whether the proposed scheme (27) leads to accurate solution, we plot approximations $\psi_{n}$, for $n=3,5$, and exact $y$ in Figure 7. The figure shows good agreement with exact solution $y$. In Figure 8 the error functions $E_{n}(x)$, for $n=3,5,7$, are plotted. In addition, numerical results for maximum absolute error $E^{(n)}$, for $n=5,8,10$, are listed in Table 4.

Example 5. Consider the linear singular two-point boundary value problem [10]

$$
\begin{gathered}
\left(x^{\alpha} y^{\prime}\right)^{\prime}=\beta x^{\alpha+\beta-2}\left(\beta x^{\beta}+\alpha+\beta-1\right) y, \quad 0<x \leq 1, \\
y(0)=1, \quad y(1)=e,
\end{gathered}
$$

with exact solution $y(x)=e^{x^{\beta}}$.

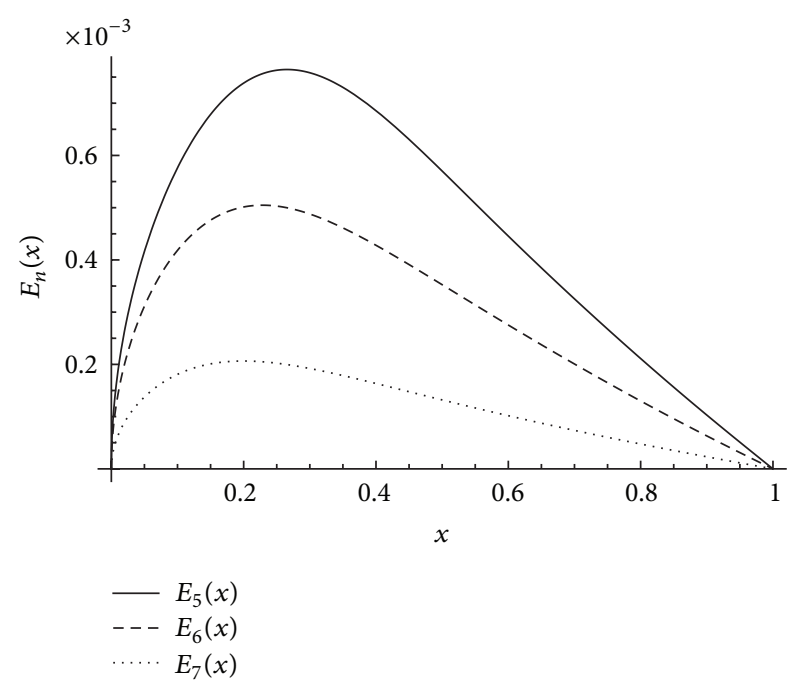

Figure 6: Error functions $E_{n}, n=5,6,7$, of Example 3 .

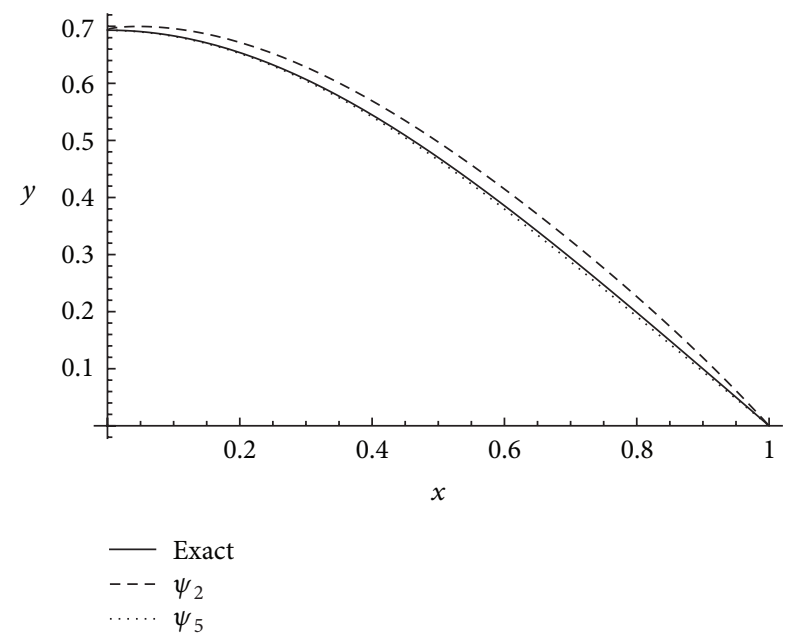

FIGURE 7: Comparison of exact solution $y$ and approximate solutions $\psi_{n}, n=2,5$, of Example 4.

We apply the recursive scheme (27) to (43), where $a=1$, $b=0, c=e$, and $d=1$, scheme (27) for (43) can be read as

$$
\begin{aligned}
& y_{0}=1, \\
& \begin{aligned}
y_{1}=(e-1) x^{1-\alpha}+x^{1-\alpha} \int_{0}^{1} x^{-\alpha} \int_{x}^{1} x^{\alpha+\beta-2} A_{0} d x d x \\
\quad-\int_{0}^{x} x^{-\alpha} \int_{x}^{1} x^{\alpha+\beta-2} A_{0} d x d x, \\
y_{n+1}=x^{1-\alpha} \int_{0}^{1} x^{-\alpha} \int_{x}^{1} x^{\alpha+\beta-2} A_{n} d x d x \\
\quad-\int_{0}^{x} x^{-\alpha} \int_{x}^{1} x^{\alpha+\beta-2} A_{n} d x d x, \quad n \geq 1,
\end{aligned}
\end{aligned}
$$

where the Adomian's polynomials are given by $A_{n}=\beta\left(\beta x^{\beta}+\right.$ $\alpha+\beta-1) y_{n}$. 


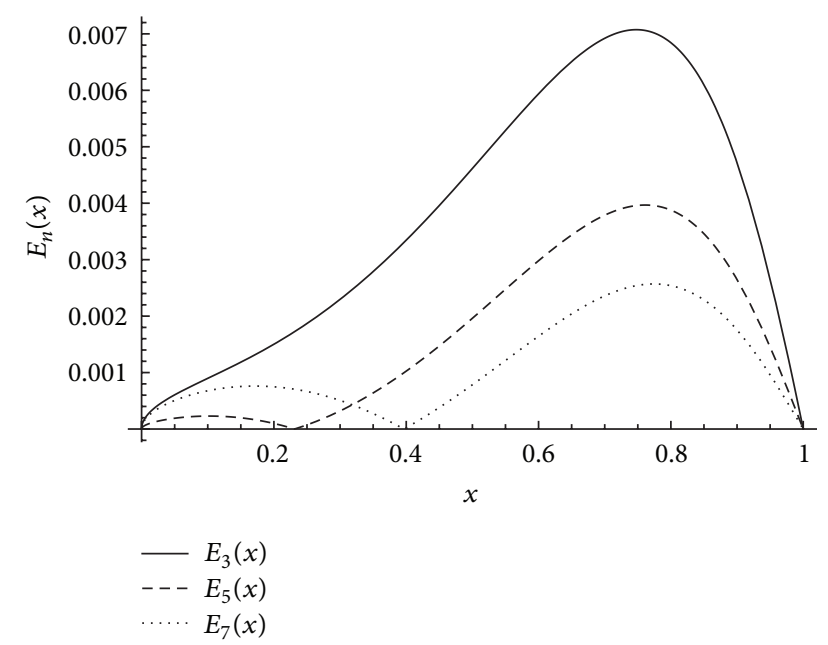

Figure 8: Error functions $E_{n}, n=5,6,7$, of Example 4.

TABLE 4: Maximum error of Example 4.

\begin{tabular}{lccc}
\hline$n$ & 5 & 8 & 10 \\
\hline$E^{(n)}$ & $6.92128 \times 10^{-3}$ & $8.25260 \times 10^{-4}$ & $2.08089 \times 10^{-4}$ \\
\hline
\end{tabular}

For $\alpha=0.25, \beta=1$ : we obtain the successive solution components $y_{n}, n>0$ using (44)

$$
\begin{aligned}
y_{0}=1 & \\
y_{1}= & 0.318282 x^{0.75}+x+0.4 x^{2} \\
y_{2}= & -0.39707 x^{0.75}+0.0454688 x^{1.75} \\
& +0.1 x^{2}+0.0578694 x^{2.75} \\
& +0.162963 x^{3}+0.0307692 x^{4} \\
\vdots &
\end{aligned}
$$

For $\alpha=0.5, \beta=1$, we have the successive solution components $y_{n}, n>0$, using (44):

$$
\begin{aligned}
y_{0}=1, & \\
y_{1}= & 0.384948 x^{0.5}+x+0.333333 x^{2} \\
y_{2}= & -0.551338 x^{0.5}+0.128316 x^{1.5} \\
& +0.166667 x^{2}+0.0769897 x^{2.5} \\
& +0.155556 x^{3}+0.0238095 x^{4}
\end{aligned}
$$

We plot approximate solutions $\psi_{n}$, for $n=1,4$, and exact solution $y$ in Figure 9. The maximum absolute errors $E^{(n)}$, for $n=5,8,10$, are displayed in Table 5 . In addition, the error functions $E_{n}(x)$, for $n=5,6,7$, are plotted in Figure 10 .

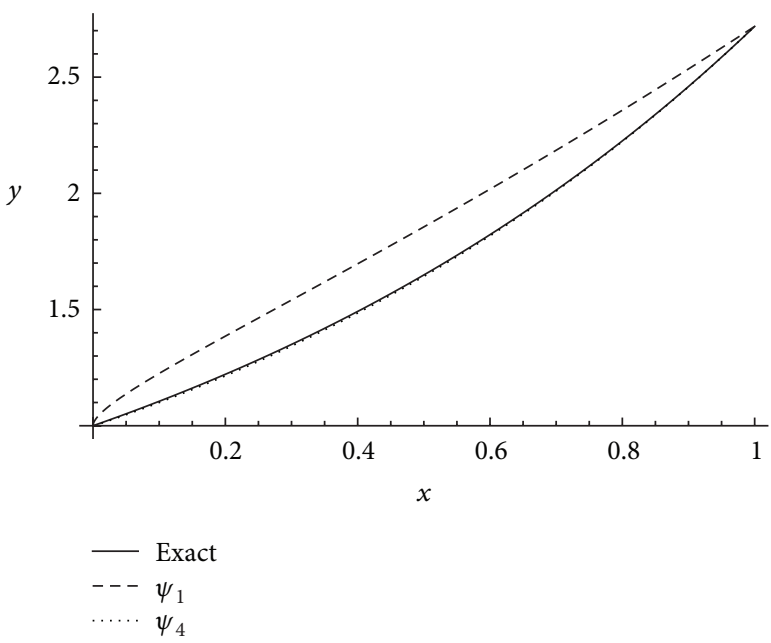

FIgURE 9: Comparison of exact solution $y$ and approximate solutions $\psi_{n}, n=1,4$, of Example 5 when $\alpha=0.5, \beta=1$.

TABLE 5: Maximum error of Example 5, when $\beta=1$.

\begin{tabular}{lccc}
\hline$\alpha$ & $E^{(5)}$ & $E^{(8)}$ & $E^{(10)}$ \\
\hline 0.25 & $1.58242 \times 10^{-4}$ & $1.24094 \times 10^{-6}$ & $4.52334 \times 10^{-8}$ \\
0.5 & $8.72985 \times 10^{-4}$ & $7.42702 \times 10^{-5}$ & $8.03711 \times 10^{-6}$ \\
\hline
\end{tabular}

Example 6. Consider the nonlinear singular two-point boundary value problem known as Thomas-Fermi equation [8]:

$$
\begin{gathered}
y^{\prime \prime}=x^{-1 / 2} y^{3 / 2}, \quad 0<x<1, \\
y(0)=1, \quad y(1)=0 .
\end{gathered}
$$

On applying scheme (27) to (47), where $a=1, b=c=0$, and $d=1$, scheme (27) for (47) becomes as follows:

$$
\begin{aligned}
y_{0}=1 & \\
y_{1}= & -x+x \int_{0}^{1} \int_{x}^{1} x^{-1 / 2} A_{0} d x d x \\
& -\int_{0}^{x} \int_{x}^{1} x^{-1 / 2} A_{0} d x d x \\
y_{n+1}= & x \int_{0}^{1} \int_{x}^{1} x^{-1 / 2} A_{n} d x d x \\
& \quad-\int_{0}^{x} \int_{x}^{1} x^{-1 / 2} A_{n} d x d x, \quad n \geq 1 .
\end{aligned}
$$

Proceeding as before, the Adomian polynomials for $f(y)=$ $y^{3 / 2}$ with $y_{0}=\ln (2)$ are given by

$$
\begin{aligned}
& A_{0}=1, \\
& A_{1}=1.5 y_{1}, \\
& A_{2}=0.375 y_{1}^{2}+1.5 y_{2}, \\
& A_{3}=-0.0625 y_{1}^{3}+0.75 y_{1} y_{2}+1.5 y_{3},
\end{aligned}
$$




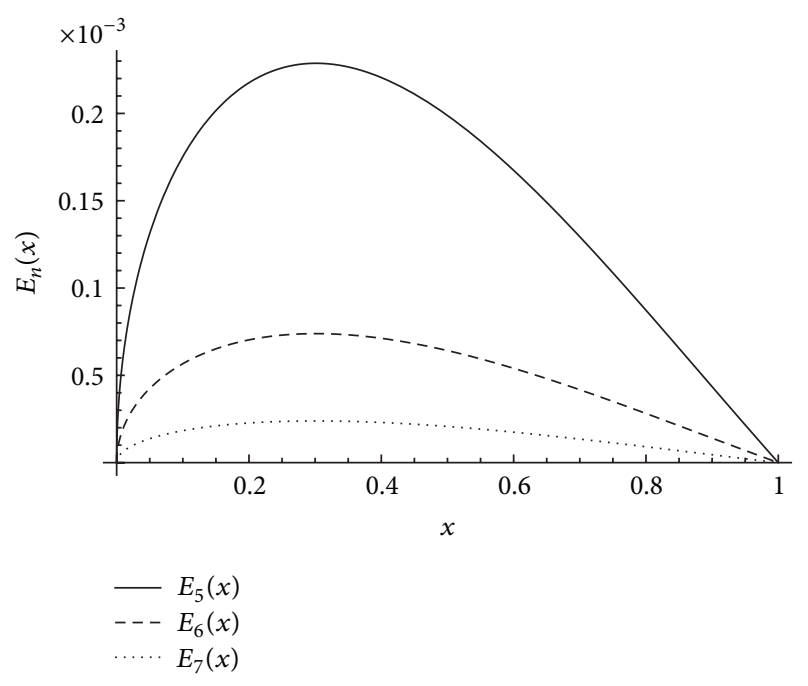

Figure 10: Error functions $E_{n}, n=5,6,7$, of Example 5 when $\alpha=$ $0.5, \beta=1$.

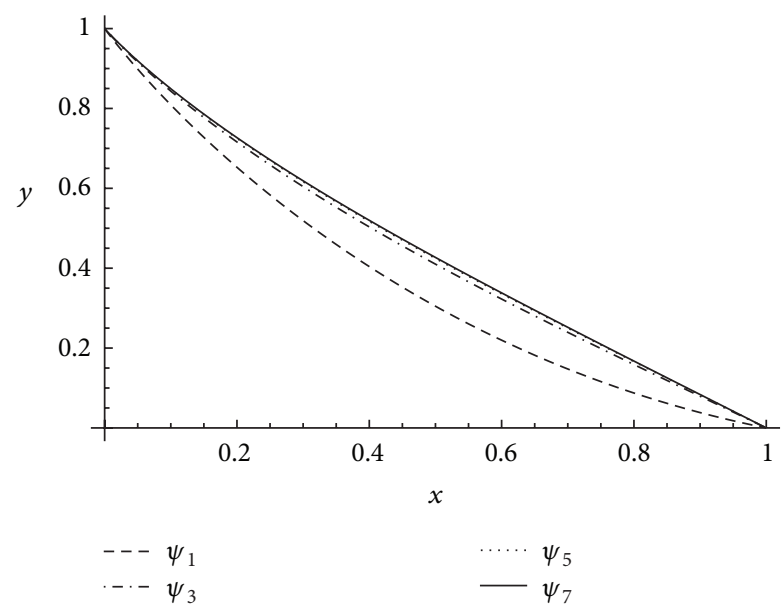

FIGURE 11: The approximate solutions $\psi_{n}, n=1,3,5,7$, of Example 6.

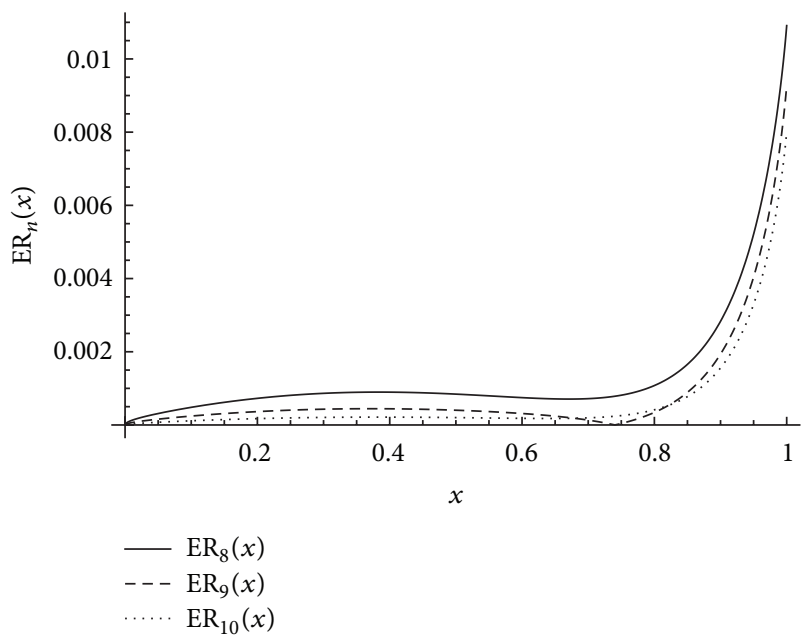

Figure 12: Error remainder function $\mathrm{ER}_{n}(x)$ for $n=8,9,10$, of Example 6.
TABLE 6: The roots of matching equations $\phi_{n}(1)=y(1)$ in $B$.

\begin{tabular}{lc}
\hline$n$ & Real roots for $B$ \\
\hline 1 & -2.333333333333 \\
2 & -1.904761904762 \\
3 & -1.886959992813 \\
4 & -1.900137008160 \\
5 & -1.904680051375 \\
6 & -1.905652841857 \\
7 & -1.905958152343 \\
8 & -1.906114487541 \\
9 & -1.906205619766 \\
10 & -1.906260891987 \\
\hline
\end{tabular}

Using (48) and (49), we have solution components $y_{n}$ of solution $y(x)$ as follows:

$$
\begin{aligned}
& y_{0}=1, \\
& y_{1}=-2.33333 x+1.33333 x^{1.5}, \\
& y_{2}= 0.6 x-0.933333 x^{2.5}+0.333333 x^{3}, \\
& y_{3}=-0.236296 x+0.24 x^{2.5}+0.233333 x^{3.5} \\
& \quad-0.311111 x^{4}+0.0740741 x^{4.5},
\end{aligned}
$$

Since the exact solution $y$ of this problem is not known, we instead investigate the error remainder function, which is a measure of how well the approximate solutions satisfy the original equation,

$$
\mathrm{ER}_{n}(x)=\psi_{n}^{\prime \prime}(x)-x^{-1 / 2} \psi_{n}^{3 / 2}(x),
$$

where the $n$-term approximate solution $\psi_{n}$ is used in place of $y$ in order to check the convergence of $\psi_{n}$ to $y$, since $\psi_{n} \rightarrow y$ as $n \rightarrow \infty$. We plot the error remainder function $\operatorname{ER}_{n}(x)$, for $n=8,9,10$, in Figure 12. From the figure we see that $\mathrm{ER}_{n}(x)$ is converging to 0 as expected. In addition, we have also plotted the approximate solutions $\psi_{n}, n=1,3,5,7$, in Figure 11. It is clear from the figure that the numerical solution converges to a certain function as the number $n$ increases.

In order to verify our numerical results, we also solve the same problem using modified ADM given by $[12,21,22]$. We apply scheme (12) to (47), scheme (12) for (47) can be read as follows:

$$
\begin{aligned}
& y_{0}=1, \\
& y_{1}=B x+\int_{0}^{x} \int_{0}^{x} x^{-1 / 2} A_{0} d x d x, \\
& y_{n+1}=\int_{0}^{x} \int_{0}^{x} x^{-1 / 2} A_{n} d x d x, \quad n \geq 1,
\end{aligned}
$$

where $B=y^{\prime}(0)$. 
TABLe 7: Comparison of numerical solutions between $\psi_{n}$ and $\phi_{n}$, for $n=4,6$.

\begin{tabular}{lcccc}
\hline$x$ & $\psi_{4}$ & $\phi_{4}$ & $\psi_{6}$ & $\phi_{6}$ \\
\hline 0 & 1 & 1 & 1 & 1 \\
0.1 & 0.8522423635 & 0.8501067213 & 0.8500236323 & 0.8495484088 \\
0.2 & 0.7323982960 & 0.7285222308 & 0.7282578059 & 0.7273830360 \\
0.3 & 0.6262858379 & 0.6212711270 & 0.6206821720 & 0.6195269347 \\
0.4 & 0.5285276286 & 0.5230884400 & 0.5220226768 & 0.5207318595 \\
0.5 & 0.4360215033 & 0.4308844855 & 0.4292273964 & 0.4279528751 \\
0.6 & 0.3467535427 & 0.3425502678 & 0.3402853521 & 0.3391664153 \\
0.7 & 0.2593472508 & 0.2565019303 & 0.2537849766 & 0.2529290646 \\
0.8 & 0.1728401288 & 0.1714556547 & 0.1687032793 & 0.1681679638 \\
0.9 & 0.0865524972 & 0.0862975946 & 0.0842902775 & 0.0840670588 \\
1 & 0 & 0 & 0 & 0 \\
\hline
\end{tabular}

TABLE 8: Comparison of numerical solutions between $\psi_{n}$ and $\phi_{n}$, for $n=8,10$.

\begin{tabular}{lcccc}
\hline$x$ & $\psi_{8}$ & $\phi_{8}$ & $\psi_{10}$ & $\phi_{10}$ \\
\hline 0 & 1 & 1 & 1 & 1 \\
0.1 & 0.8495969286 & 0.8495016788 & 0.8495046727 & 0.8494868590 \\
0.2 & 0.7274614883 & 0.7272876062 & 0.7272890033 & 0.7272573380 \\
0.3 & 0.6196064297 & 0.6193802967 & 0.6193729507 & 0.6193337312 \\
0.4 & 0.5207781835 & 0.5205320369 & 0.5205073481 & 0.5204682806 \\
0.5 & 0.4279329340 & 0.4277006140 & 0.4276499517 & 0.4276191499 \\
0.6 & 0.3390569614 & 0.3388691705 & 0.3387861666 & 0.3387709561 \\
0.7 & 0.2527284963 & 0.2526072061 & 0.2524919010 & 0.2524970231 \\
0.8 & 0.1679120917 & 0.1678634079 & 0.1677297778 & 0.1677541091 \\
0.9 & 0.0838498011 & 0.0838543745 & 0.0837432823 & 0.0837736317 \\
1 & 0 & 0 & 0 & 0 \\
\hline
\end{tabular}

Using (52) and (49), we have solution components $y_{n}$ of solution $y(x)$ as follows:

$$
\begin{aligned}
y_{0}= & 1, \\
y_{1}= & B x+1.33333 x^{1.5} \\
y_{2}= & 0.4 B x^{2.5}+0.333333 x^{3} \\
y_{3}= & 0.0428571 B^{2} x^{3.5}+0.133333 B x^{4} \\
& +0.0740741 x^{4.5}
\end{aligned}
$$

and the approximate solutions $\phi_{n}=y_{0}+y_{1}+y_{2}+\cdots+y_{n}$ given by

$$
\begin{aligned}
\phi_{1}(x)=1 & +B x+1.33333 x^{1.5} \\
\phi_{2}(x)=1 & +B x+1.33333 x^{1.5}+0.4 B x^{2.5} \\
& +0.333333 x^{3} \\
\phi_{3}(x)=1 & +B x+1.33333 x^{1.5}+0.4 B x^{2.5} \\
& +0.333333 x^{3}+0.0428571 B^{2} x^{3.5} \\
+ & 0.133333 B x^{4}+0.0740741 x^{4.5}
\end{aligned}
$$


to obtain approximate solutions, and we have also shown graphically that these approximate solutions are almost identical to the analytic solution. Another major advantage of the proposed method is that it does not require the computation of undetermined coefficients. However, most of the previous recursive schemes using $\mathrm{ADM}$ or MDAM do require the computation of undetermined coefficients which increases computational work (see for more details [3, 14, 15, 22, 23]). The proposed method provides a reliable technique which requires less work compared to the traditional techniques such as finite difference method, cubic spline method, and standard ADM or MADM. The numerical results of the examples are presented, and only a few terms are required to obtain accurate solutions. By comparing the results with other existing methods, it has been proved that the proposed method is a powerful method for solving singular two-point boundary value problems.

\section{References}

[1] M. M. Chawla and C. P. Katti, "Finite difference methods and their convergence for a class of singular two point boundary value problems," Numerische Mathematik, vol. 39, no. 3, pp. 341350, 1982.

[2] M. Kumar, "A fourth-order finite difference method for a class of singular two-point boundary value problems," Applied Mathematics and Computation, vol. 133, no. 2-3, pp. 539-545, 2002.

[3] M. Inc and D. J. Evans, "The decomposition method for solving of a class of singular two-point boundary value problems," International Journal of Computer Mathematics, vol. 80, no. 7, pp. 869-882, 2003.

[4] Z. Cen, "Numerical study for a class of singular two-point boundary value problems using Green's functions," Applied Mathematics and Computation, vol. 183, no. 1, pp. 10-16, 2006.

[5] M. Kumar and T. Aziz, "A uniform mesh finite difference method for a class of singular two-point boundary value problems," Applied Mathematics and Computation, vol. 180, no. 1, pp. 173-177, 2006.

[6] S. H. Lin, "Oxygen diffusion in a spherical cell with nonlinear oxygen uptake kinetics," Journal of Theoretical Biology, vol. 60, no. 2, pp. 449-457, 1976.

[7] B. F. Gray, "The distribution of heat sources in the human head-theoretical consideration," Journal of Theoretical Biology, vol. 82, no. 3, pp. 473-476, 1980.

[8] G. Adomian, "Solution of the Thomas-Fermi equation," Applied Mathematics Letters, vol. 11, no. 3, pp. 131-133, 1998.

[9] P. Jamet, "On the convergence of finite-difference approximations to one-dimensional singular boundary-value problems," Numerische Mathematik, vol. 14, no. 4, pp. 355-378, 1970.

[10] T. Aziz and M. Kumar, "A fourth-order finite-difference method based on non-uniform mesh for a class of singular twopoint boundary value problems," Journal of Computational and Applied Mathematics, vol. 136, no. 1-2, pp. 337-342, 2001.

[11] M. Kumar, "A new finite difference method for a class of singular two-point boundary value problems," Applied Mathematics and Computation, vol. 143, no. 2-3, pp. 551-557, 2003.

[12] M. Inc, M. Ergüt, and Y. Cherruault, "A different approach for solving singular two-point boundary value problems," Kybernetes, vol. 34, no. 7-8, pp. 934-940, 2005.

[13] A. Ebaid, "A new analytical and numerical treatment for singular two-point boundary value problems via the Adomian decomposition method," Journal of Computational and Applied Mathematics, vol. 235, no. 8, pp. 1914-1924, 2011.

[14] S. A. Khuri and A. Sayfy, "A novel approach for the solution of a class of singular boundary value problems arising in physiology," Mathematical and Computer Modelling, vol. 52, no. 3-4, pp. 626-636, 2010.

[15] M. Kumar and N. Singh, "Modified Adomian decomposition method and computer implementation for solving singular boundary value problems arising in various physical problems," Computers and Chemical Engineering, vol. 34, no. 11, pp. 1750$1760,2010$.

[16] B. Jang, "Two-point boundary value problems by the extended Adomian decomposition method," Journal of Computational and Applied Mathematics, vol. 219, no. 1, pp. 253-262, 2008.

[17] G. Adomian and R. Rach, "Inversion of nonlinear stochastic operators," Journal of Mathematical Analysis and Applications, vol. 91, no. 1, pp. 39-46, 1983.

[18] G. Adomian and R. Rach, "A new algorithm for matching boundary conditions in decomposition solutions," Applied Mathematics and Computation, vol. 57, no. 1, pp. 61-68, 1993.

[19] G. Adomian and R. Rach, "Modified decomposition solution of linear and nonlinear boundary-value problems," Nonlinear Analysis: Theory, Methods \& Applications, vol. 23, no. 5, pp. 615619, 1994.

[20] G. Adomian, Solving Frontier Problems of Physics: The Decomposition Method, Kluwer Academic Publishers, 1994.

[21] A. M. Wazwaz, "Approximate solutions to boundary value problems of higher order by the modified decomposition method," Computers and Mathematics with Applications, vol. 40, no. 6-7, pp. 679-691, 2000.

[22] A. M. Wazwaz, "A reliable algorithm for obtaining positive solutions for nonlinear boundary value problems," Computers and Mathematics with Applications, vol. 41, no. 10-11, pp. 12371244, 2001.

[23] M. Benabidallah and Y. Cherruault, "Application of the Adomian method for solving a class of boundary problems," Kybernetes, vol. 33, no. 1, pp. 118-132, 2004.

[24] A. M. Wazwaz, "A new method for solving singular initial value problems in the second-order ordinary differential equations," Applied Mathematics and Computation, vol. 128, no. 1, pp. 45-57, 2002.

[25] J. Duan and R. Rach, "A new modification of the Adomian decomposition method for solving boundary value problems for higher order nonlinear differential equations," Applied Mathematics and Computation, vol. 218, no. 8, pp. 4090-4118, 2011.

[26] K. Abbaoui and Y. Cherruault, "Convergence of Adomian's method applied to differential equations," Computers and Mathematics with Applications, vol. 28, no. 5, pp. 103-109, 1994.

[27] Y. Cherruault, “Convergence of Adomian's method," Kybernetes, vol. 18, no. 2, pp. 31-38, 1989.

[28] M. M. Hosseini and H. Nasabzadeh, "On the convergence of Adomian decomposition method," Applied Mathematics and Computation, vol. 182, no. 1, pp. 536-543, 2006. 


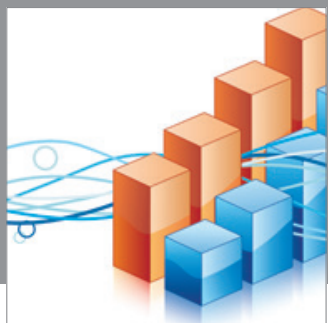

Advances in

Operations Research

mansans

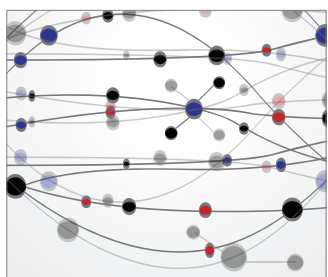

The Scientific World Journal
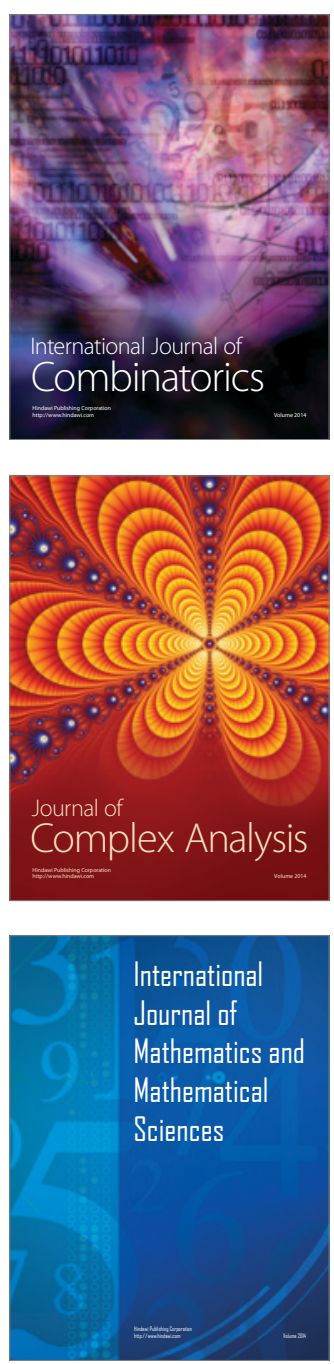
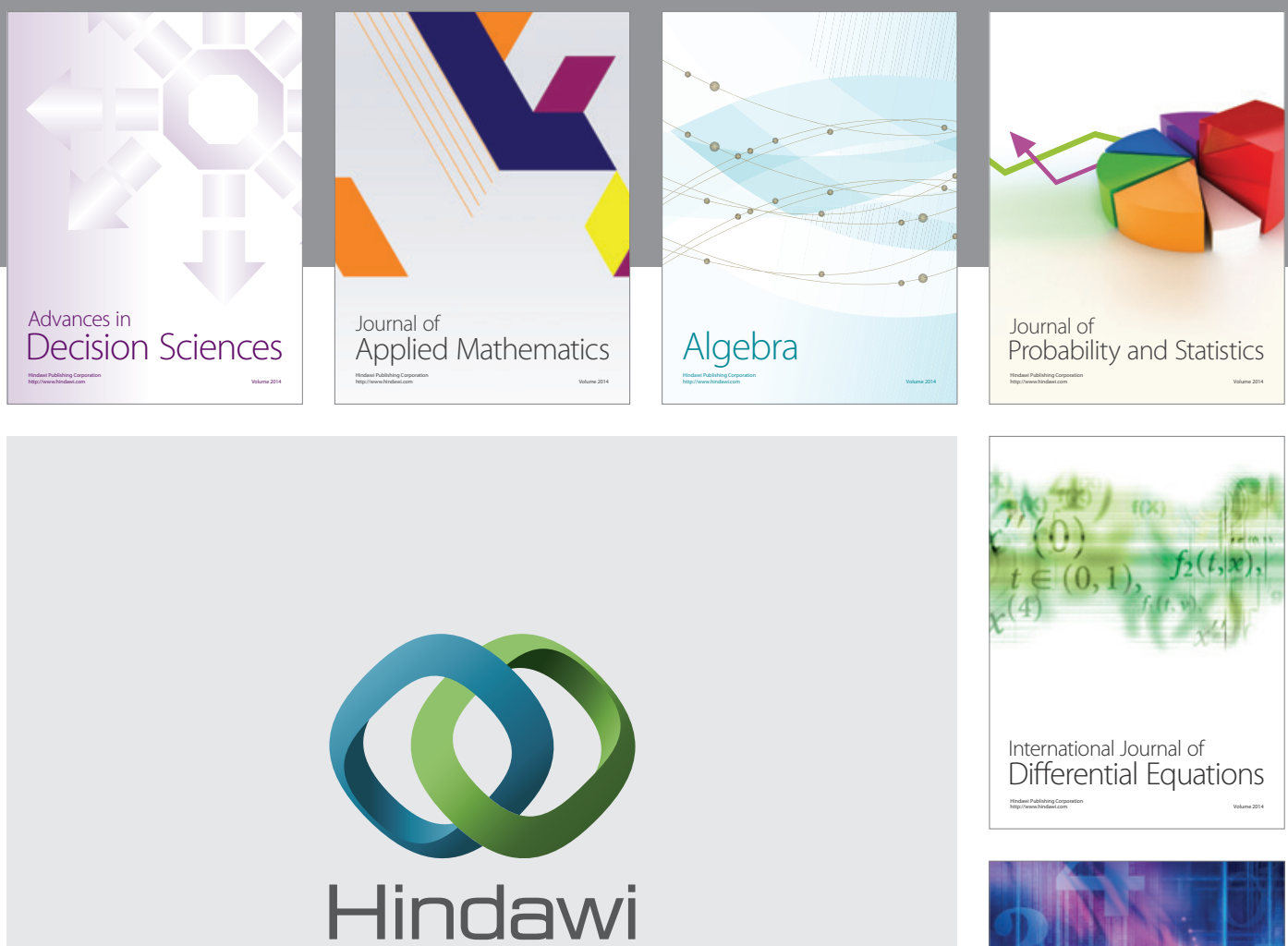

Submit your manuscripts at http://www.hindawi.com
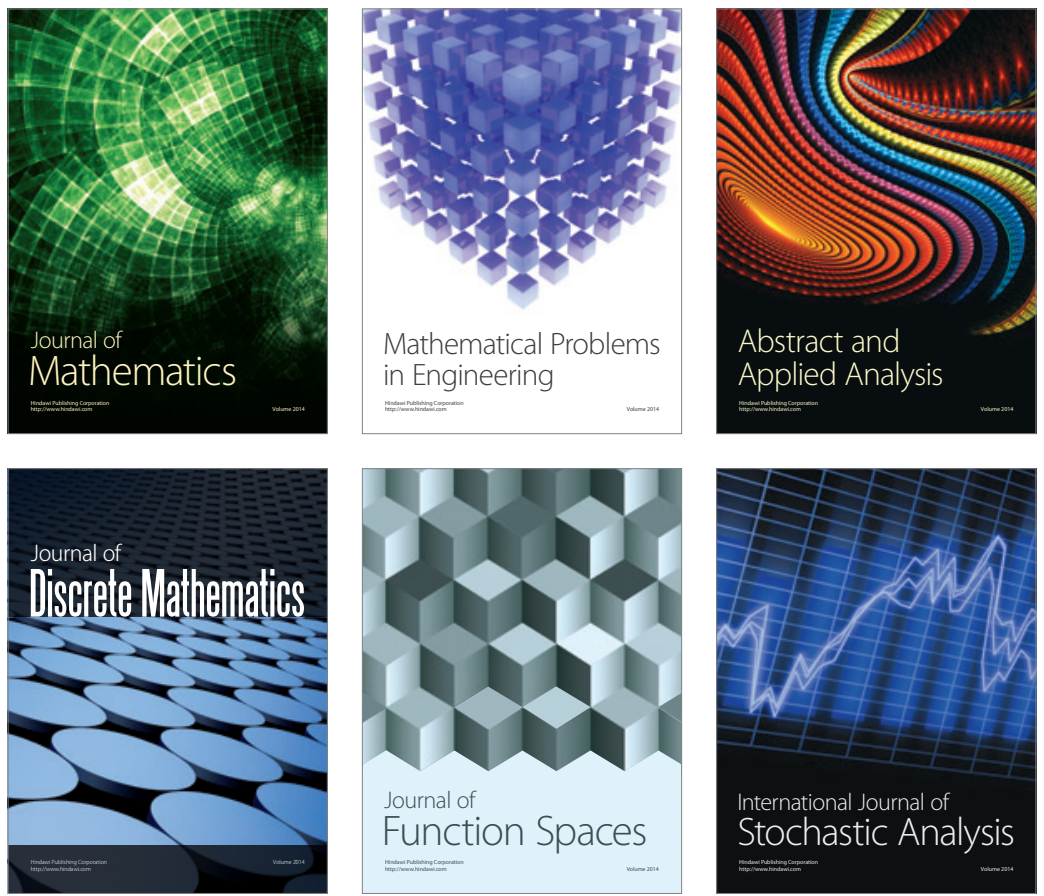

Journal of

Function Spaces

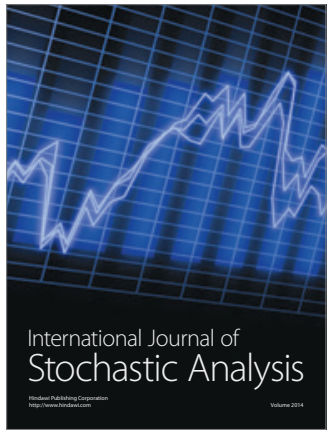

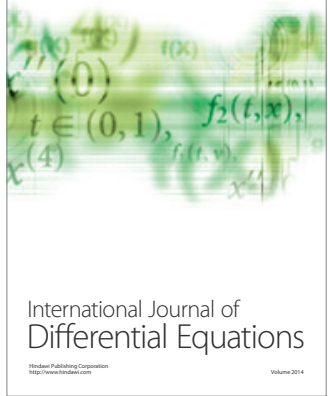
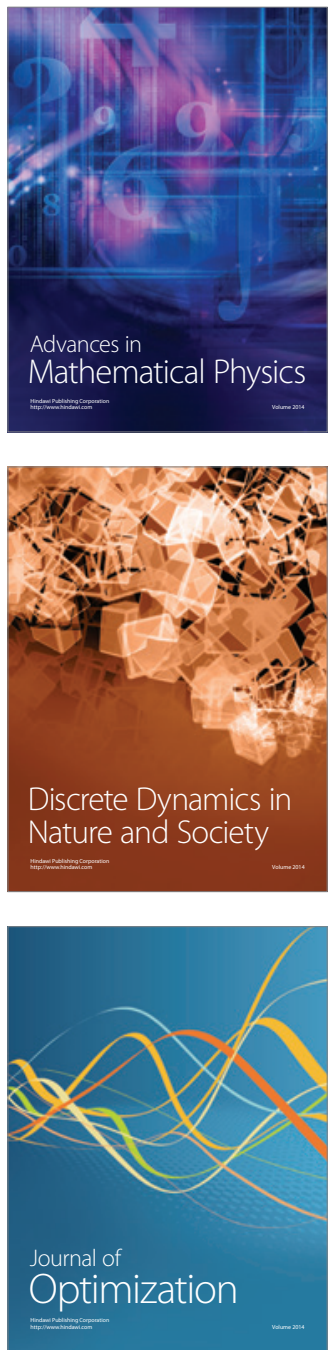